\title{
Atomistic Simulation of Field Enhanced Oxidation of Al (100) Beyond the Mott Potential
}

\section{Citation}

Sankaranarayanan, Subramanian K. R. S., Efthimios Kaxiras, and Shriram Ramanathan. 2009. "Atomistic Simulation of Field Enhanced Oxidation of Al (100) Beyond the Mott Potential."

Physical Review Letters 102 (9). https://doi.org/10.1103/physrevlett.102.095504.

\section{Permanent link}

http://nrs.harvard.edu/urn-3:HUL.InstRepos:41384119

\section{Terms of Use}

This article was downloaded from Harvard University's DASH repository, and is made available under the terms and conditions applicable to Other Posted Material, as set forth at http:// nrs.harvard.edu/urn-3:HUL.InstRepos:dash.current.terms-of-use\#LAA

\section{Share Your Story}

The Harvard community has made this article openly available.

Please share how this access benefits you. Submit a story. 


\title{
Atomistic Simulation of Field Enhanced Oxidation of Al (100) Beyond the Mott Potential
}

\author{
Subramanian K. R. S. Sankaranarayanan, Efthimios Kaxiras, and Shriram Ramanathan* \\ Harvard School of Engineering and Applied Sciences, Harvard University, Cambridge, Massachusetts 02138, USA
}

(Received 18 December 2008; published 6 March 2009)

\begin{abstract}
Molecular dynamics simulations employing dynamic charge transfer between atoms indicate a significantly enhanced rate of $\mathrm{Al}(100)$ oxidation by $\mathrm{O}_{2}$ and $\mathrm{O}$ at $300 \mathrm{~K}$ in the presence of an electric field. Increasing the electric field $\left(\sim 10^{7} \mathrm{~V} / \mathrm{cm}\right)$ drives the surface chemisorbed oxygen to the vacancy sites in the oxide interior leading to dramatic density and stoichiometry improvements of the grown ultrathin oxide film. The associated oxidation kinetics enhancement due to the applied electric field is postulated to arise from the activation barrier lowering at electrostatic potentials approaching the Mott potential and beyond, leading to a dramatically increased ion migration through oxide film. The results are of significance to understanding mechanisms of early stage oxide growth as well as technologies utilizing ultrathin oxides.
\end{abstract}

DOI: 10.1103/PhysRevLett.102.095504

Ultrathin aluminum oxide layers find applications as tunnel barriers in superconducting Josephson junctions and magnetic tunnel junctions, passivation films to protect against pitting corrosion, gate dielectrics in advanced transistors, and in heterogeneous catalyses [1-3]. In particular, the stoichiometry and microstructure of the oxide film play a prominent role in determining its electrical, optical, thermal, chemical, and mechanical properties. For example, nonstoichiometric alumina can have a much smaller band gap compared with that of bulk stoichiometric alumina $(\sim 8-9 \mathrm{eV})[1]$.

Recent experimental investigations on oxide films grown under the influence of electric fields as well as under photon exposure have shown dramatic improvement in oxide quality $[1,3,4]$. Electron stimulated oxidation of $\mathrm{Al}$ (111) resulted in significantly enhanced oxidation kinetics at low temperatures [5]. Similar effects were observed for photon-assisted oxide growth [6-8]. In both cases, the rate of oxygen incorporation was dramatically enhanced compared to natural oxidation leading to a significant improvement in the quality of oxide films [6,7]. In one of our previous investigations, the oxide stoichiometry and density of native alumina layers were dramatically improved by minutes-long exposure to photon irradiation, leading to a 34\% increase in its corrosion resistance [9].

In case of electric-field assisted oxide growth, mechanistic understanding of the microscopic processes during the initial and subsequent stages of oxide-film growth is still unclear. While there are some limited experimental data on the initial oxidation rates of different metals under the influence of external fields, there are presently no theoretical models to quantitatively explain the observed enhancement at the atomistic scale [10]. In the case of electron enhanced oxidation, most of the experimental kinetic data have been explained on the basis of the Mott-Cabrera theory, which postulates that the electrostatic field formed across a growing oxide film promotes ion migration-the limiting step for mass transport in oxida-
PACS numbers: 81.65.Mq, 68.47.Gh, 81.05.Bx, 82.20.Wt

tion, leading to a rapid and more uniform oxide-film growth [11]. This basic model however does not consider the effects arising from the defects or disorder in the oxide layer and also does not take into account the atomistic processes occurring at the metal surface, in the developing oxide film, and at the metal-oxide and oxide-gas interfaces.

In this Letter, we report the first variable charge molecular dynamics simulation investigating the initial oxidation kinetics and ultrathin oxide growth under the influence of electric fields. Mechanistic details of the electric field assisted oxidation kinetics and oxide growth at atomistic scale is elucidated. The structural and morphological differences in the growing oxide film are evaluated using dynamical correlation functions. The evolution of charges, self-limiting oxide thicknesses and atomic diffusivities for the two oxidizing conditions (atomic vs molecular) under varying electric fields are used to clearly explain the experimentally observed enhancement in the oxidation kinetics and the quality of the grown oxide film.

The molecular dynamic (MD) simulations employ a potential model that allows for variable and dynamic charge transfer between atoms and is capable of treating both metallic and ceramic systems as well as bond formation and bond breakage involved in oxidation processes [12-14]. It has been used to extensively study the oxidation of $\mathrm{Al}$ surfaces. Moreover, previously reported simulations of low index surfaces of $\alpha$ alumina have shown good agreement with those by first principles density functional theory calculations $[13,14]$. Here, we consider the effect of the electric field $E(V / m)$ on these simulations. The electric field contributes an additional energy arising from the charges on the ions:

$$
U_{q, \mathrm{ext}}=-\sum_{i}^{N} q_{i} r_{i} . E .
$$

The charges on the atoms are obtained dynamically using the charge relaxation procedure which minimizes the electrostatic energy subject to the electro-neutrality 
principle. Typical experimentally reported values of the breakdown voltage of bulk alumina correspond to 3-15 MV/cm [15]. Hence, the applied electric field was varied from 1 to $15 \mathrm{MV} / \mathrm{cm}$ to study the kinetics of field induced oxidation.

The setup of the oxidation simulations are as follows: A slab of fcc $\mathrm{Al}$ containing approximately 1000 atoms with exposed surface dimensions $\left(20 \times 20 \AA^{2}\right)$ was formed from an fcc unit cell. The Al (100) surface was then generated by artificially increasing the $x$ direction and introducing two vacuum slabs on each side of the metal substrate. This unit cell was repeated infinitely though 3D space by applying periodic boundary conditions. The oxidation of the $\mathrm{Al}$ substrate is initiated by introducing either $100 \mathrm{O}$ or $50 \mathrm{O}_{2}$ molecules in the vacuum slab with their $x, y$, and $z$ positions chosen randomly. The oxygen number density is maintained constant at approximately $0.0025 / \AA^{3}$ in both the cases. The equations of motion are integrated with time steps $\Delta t=1 \mathrm{fs}$ for both short range and long range forces. The atomic charges were updated every 100 time steps such that the electrostatic energy is minimized subject to the constraint of electro-neutrality. The MD simulations were stopped when the limiting thickness of the growing oxide film is reached $[16,17]$.

Figure 1 shows the influence of the electric field on the evolution of the oxide-film thickness for $\mathrm{Al}$ (100) oxidation up to its limiting thickness for atomic and natural oxidation. The oxide film shows an initial rapid increase followed by a slower growth phase. The oxidation kinetics at $300 \mathrm{~K}$ is significantly enhanced in the presence of the electric field $\left(\sim 10^{7} \mathrm{~V} / \mathrm{cm}\right)$. Increasing the electric field from 1 to $15 \mathrm{MV} / \mathrm{cm}$ resulted in faster kinetics and an earlier onset of the slower growth phase. The self-limiting thickness of the oxide film formed in case of molecular oxidation without the electric-field effect corresponds to $\sim 16 \AA$. A significant increase in self-limiting thickness from $\sim 17 \AA$ at $1 \mathrm{MV} / \mathrm{cm}$ to $\sim 23 \AA$ at $15 \mathrm{MV} / \mathrm{cm}$ was observed. The range of limiting thicknesses agrees well with the experimental values by Popova et al. [3]. The limiting thickness for atomic oxidation represents a $40 \%$ increase over natural oxidation in the absence of the electric field. Similarly, the application of the electric field in the $10-15 \mathrm{MV} / \mathrm{cm}$ range resulted in a significant increase in the rate of oxide growth for atomic oxidation. The simulated limiting thickness increased from approximately $24 \AA$ at $1 \mathrm{MV} / \mathrm{cm}$ to $\sim 34 \AA$ at $15 \mathrm{MV} / \mathrm{cm}$. To the best of our knowledge, there are no available experimental studies on the atomic oxidation of the $\mathrm{Al}$ (100) surface. However, the enhancement in oxidation kinetics as well as selflimiting thickness observed in our simulations is consistent with that observed experimentally for low temperature field-assisted oxidation of the $\mathrm{Al}$ (111) surface by Popova et al. and Zhukov et al. $[3,4]$.

The oxide scale formed is amorphous in nature. To the best of our knowledge, the differences between the amorphous oxide scale formed under various simulated electric

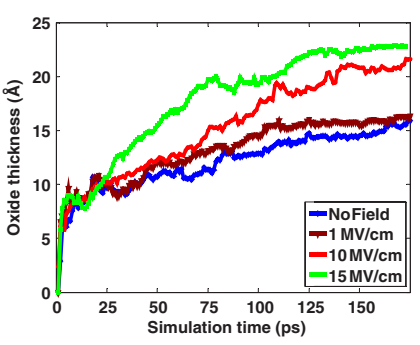

(a)

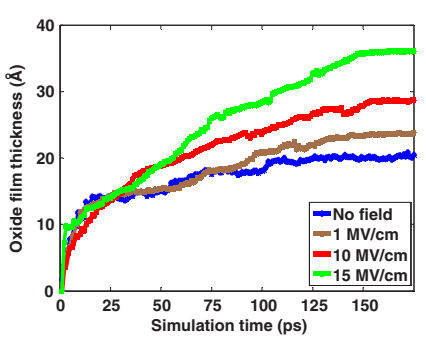

(b)
FIG. 1 (color online). Variation of oxide-film thickness with oxidation time in (a) $\mathrm{O}_{2}$ and (b) the $\mathrm{O}$ environment at room temperature. The electric field strength was varied from 1 to $15 \mathrm{MV} / \mathrm{cm}$.

fields have not been reported yet. The position of the first peak in the various calculated pair distribution function (PDF) (example Fig. 2) gave the respective bond lengths under varying electric fields, which are shown in Table I. Figure 2(a) indicates that the Al-O peak shifted from $1.90 \AA$ in the case of natural oxidation to $1.77 \AA$ for the $15 \mathrm{MV} / \mathrm{cm}$ applied electric field. The shortening of bond length is attributed to the increased oxide density in the case of electric-field assisted oxide growth. The corresponding coordination numbers for $\mathrm{Al}$ in the two cases [obtained by integrating the $\mathrm{g}(\mathrm{r})$ for $\mathrm{Al}-\mathrm{O}$ up to $2.5 \AA$ ] are 3.6 for natural oxidation and 4.7 for $15 \mathrm{MV} / \mathrm{cm}$, respectively. The PDF for O-O also showed similar shifts in the peak positions related to the change in the relative $\mathrm{Al}$ and $\mathrm{O}$ densities upon applying the electric field as shown in Table I.

The calculated $\mathrm{O}-\mathrm{Al}-\mathrm{O}$ bond angle distribution in the interior of the native oxide scale for various applied electric fields in Fig. 2 shows peaks at $90^{\circ}$ and $109^{\circ}$. These two peaks in the oxide region indicate mixed octahedral, $\mathrm{Al}\left(\mathrm{O}_{1 / 6}\right)_{6}$, and tetrahedral, $\mathrm{Al}\left(\mathrm{O}_{1 / 4}\right)_{4}$, configurations, respectively. These peaks shift toward slightly smaller angles as we increase the electric field from 0 to $15 \mathrm{MV} / \mathrm{cm}$, reflecting the increase in oxygen density. Furthermore, Fig. 2 shows that the fraction of the tetrahedrally and

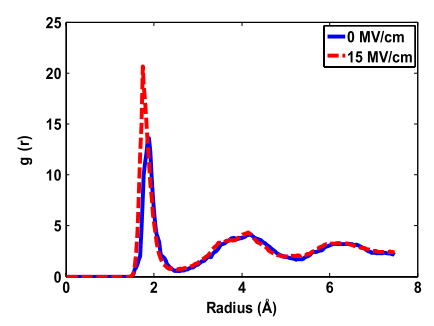

(a)

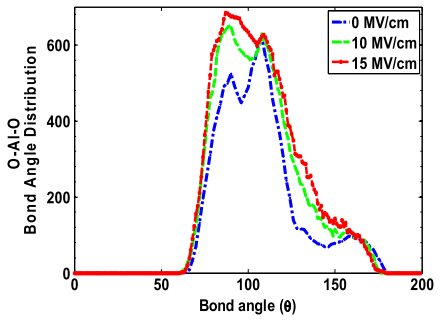

FIG. 2 (color online). (a) Pair distribution function Al-O in the $\mathrm{Al} / \mathrm{O}$ system taken at $100 \mathrm{ps}$ of exposure time for an applied electric field of $15 \mathrm{MV} / \mathrm{cm}$ for the case of natural oxidation. The calculated bond lengths are summarized in Table I for various values of the applied electric field (b) O-Al-O bond angle distribution in the grown oxide film for various values of the applied electric field. 
TABLE I. Effect of the applied electric field on the calculated bond lengths $(\AA)$ in the oxide film for natural oxidation of aluminum substrate. The electric field was varied from 0 to $15 \mathrm{MV} / \mathrm{cm}$.

\begin{tabular}{ccccc}
\hline $\begin{array}{c}\text { Bond length } \\
(\AA)\end{array}$ & Natural & $1 \mathrm{MV} / \mathrm{cm}$ & $10 \mathrm{MV} / \mathrm{cm}$ & $15 \mathrm{MV} / \mathrm{cm}$ \\
\hline $\mathrm{Al}-\mathrm{Al}$ & 2.90 & 2.90 & 2.90 & 2.90 \\
$\mathrm{Al}-\mathrm{O}$ & 1.90 & 1.90 & 1.83 & 1.77 \\
$\mathrm{O}-\mathrm{O}$ & 3.30 & 3.10 & 3.10 & 3.0 \\
\hline \hline
\end{tabular}

octahedrally coordinated configurations change with the applied electric field. The increase in the electric field results in a corresponding increase in the fraction of octahedrally coordinated atoms as suggested by the relative peak intensities at $\sim 90^{\circ}$ and $109^{\circ}$. Thus, the simulation results indicate that the coordination number of the elementary unit of the oxide formed increases with the applied electric field. Typically, the proportion of the $\mathrm{AlO}_{4}$ tetrahedron and $\mathrm{AlO}_{6}$ octahedron is dictated by the oxide density, with the $\mathrm{AlO}_{6}$ fraction becoming important as the oxide density increases. This suggests that the oxide scale formed in the presence of the electric field is denser.

Our analysis of the oxygen density profiles for natural oxidation without the electric field indicates significantly lower densities at the metal-oxide interface and higher densities close to the oxide-gas interface. There is a gradation in oxygen densities in the interior of the oxide scale, with a significant drop as we approach the metal-oxide interface. Thus, the oxide produced by natural oxidation appears to be substoichiometric and oxygen deficient, especially in the oxide interior and the metal-oxide interface. As we increase the electric field, the oxygen densities at both the oxide gas and metal-oxide interface become significantly higher, which can be attributed to increased anion migration into the oxide film. This agrees well with the experimental investigation of Chang et al., who reported the formation of an $18 \%$ denser oxide resulting from both improvements in oxygen stoichiometry as well as structural changes in case of UV assisted oxidation of pure $\mathrm{Al}$ substrates [1,9].

The simulated average composition expressed as the $\mathrm{O} / \mathrm{Al}$ ratios increase with time during the fast oxidation stage and attain a more or less constant value in the second slow oxidation stage, when the oxide film reaches its selflimiting thickness. In the case of natural oxidation without any externally applied electric field, the oxide film has an overall nonstoichiometric composition, which varies from 1.27 at the oxide-gas interface to 1.05 at the oxide-metal interface, as shown in Table II. Our simulation results indicate a gradation of oxygen stoichiometry across the oxide thickness such that the oxygen density is low at the metal-oxide interface and higher at the oxide-gas interface. This relative enrichment of the cations in regions close to the oxide-metal interface has also been observed in the case of natural oxidation of other metal substrate-oxide-
TABLE II. Effect of the applied electric field on the calculated oxide stoichiometry across the oxide film at $300 \mathrm{~K}$ for the case of natural oxidation. The electric field was varied from 0 to $15 \mathrm{MV} / \mathrm{cm}$.

\begin{tabular}{lccc}
\hline \hline $\mathrm{O} / \mathrm{Al}$ & Oxide gas & Oxide interior & Oxide metal \\
\hline Natural & 1.27 & 1.16 & 1.05 \\
$1 \mathrm{MV} / \mathrm{cm}$ & 1.29 & 1.18 & 1.05 \\
$10 \mathrm{MV} / \mathrm{cm}$ & 1.34 & 1.29 & 1.10 \\
$15 \mathrm{MV} / \mathrm{cm}$ & 1.40 & 1.37 & 1.18 \\
\hline \hline
\end{tabular}

film systems [18]. When the electric field is increased from no field to $15 \mathrm{MV} / \mathrm{cm}$ for natural oxidation, the $\mathrm{O} / \mathrm{Al}$ ratios increase from 1.27 to $1.40,1.16$ to 1.37 , and 1.05 to 1.18 at the oxide-gas, oxide-interior, and oxide-metal interfaces, respectively. Similar stoichiometry improvements from 1.37 to $1.42,1.35$ to 1.39 , and 1.30 to 1.32 at the oxide-gas, oxide-interior, and oxide-metal interfaces, respectively, are also observed in case of atomic oxidation of $\mathrm{Al}$ from no field to $15 \mathrm{MV} / \mathrm{cm}$. These results are in excellent agreement with the experimental observations of electric-field enhanced room temperature $\mathrm{Al}$ oxidation [1].

A possible mechanism for the observed increase in the oxidation kinetics and oxide film quality in the presence of the electric field involves the transformation of chemisorbed $\mathrm{O}$ species on the surface to the oxidic species. To estimate the relative populations of the two species, we compare the power spectra obtained as Fourier transformation of the velocity autocorrelation function calculated from the MD simulated dynamic trajectories of the atoms in the oxide-gas interface in the presence and absence of the electric field. The vibrational spectra shown in Fig. 3(a) show two distinct bands at $\sim 600$ and $800 \mathrm{~cm}^{-1}$. Previously reported experimental investigations (highresolution electron-energy-loss spectroscopy spectra) suggest that the modes at 590 and $820 \mathrm{~cm}^{-1}$ correspond to

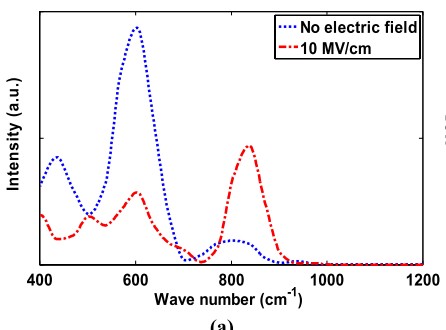

(a)

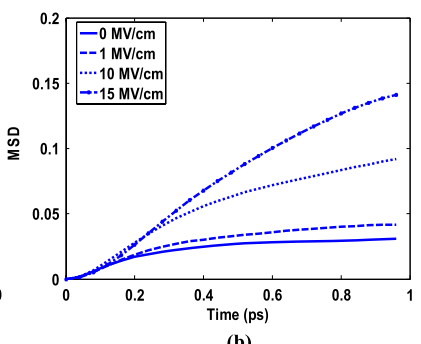

(b)
FIG. 3 (color online). (a) Vibrational spectra obtained by Fourier transformation of the velocity autocorrelation function obtained at $10 \mathrm{ps}$ of exposure time. Low frequency mode near $600 \mathrm{~cm}^{-1}$ is reflective of the chemisorbed $\mathrm{O}$ atoms, whereas the higher frequency mode near $820 \mathrm{~cm}^{-1}$ corresponds to the oxidic species (b) mean square displacement (MSD) representing the inward diffusion of oxygen atoms located at the oxide-gas interface for various values of the applied electric field. MSD shown is at $40 \mathrm{ps}$ of exposure time for natural oxidation of the $\mathrm{Al}$ (100) substrate. 
chemisorbed and oxidic species, respectively [5]. Similar frequencies were observed by Erksine and Strong and were attributed to $\mathrm{O}-\mathrm{Al}$ surface stretching motion and $\mathrm{O}-\mathrm{Al}$ subsurface stretching motion, respectively [19]. Vibrational spectra for the initial phase natural oxidation of $\mathrm{Al}$ (100) at $300 \mathrm{~K}$ suggests the domination of the low frequency mode at $\sim 600 \mathrm{~cm}^{-1}$ indicating the preferential population of surface chemisorbed $\mathrm{O}$ species. The presence of the electric field leads to a dramatic enhancement in the intensity of the high frequency mode at $800 \mathrm{~cm}^{-1}$ suggesting an increased transformation of the chemisorbed $\mathrm{O}$ species into oxidic species. The simulation results are thus consistent with the proposed mechanism where electron bombardment was experimentally found to drive the surface chemisorbed oxygen atoms into the vacancy sites, leading to enhanced oxygen uptake and dramatically improved oxygen density and stoichiometry [2].

Additionally, we compare the shell based mean square displacement for the $\mathrm{O}$ atoms located at the oxide-gas interface for various applied electric fields as shown in Fig. 3(b). Increasing the electric field leads to a significant increase in the diffusivity of the oxygen atoms into the substrate. In accordance with the Cabrera-Mott theory for low temperature oxidation, the presence of the electric field leads to a decrease in the activation energy barrier for ion migration [11]. Electron transfer from metal to oxygen results in the establishment of an electrostatic potential across the oxide film termed as the Mott potential $\left(V_{M}\right)$ and the electric field produced across the oxide-film thickness controls the ion migration rate and thereby the film growth. The electrostatic potential in the outer part of the oxide film in the case of thermal oxidation decreases linearly with the oxide-film thickness. Increasing the electrostatic potential through the externally applied electric field leads to artificial stimulation of the ion diffusion, allowing for higher film growth than the saturation limit obtained using thermal oxidation. Typical reported values of the Mott potential for aluminum oxidation corresponds to -1.8 to $-2 \mathrm{~V}$ [11]. In this work, we observe a dramatic enhancement in oxidation kinetics at applied fields of $10-15 \mathrm{MV} / \mathrm{cm}$, when the self-limiting thickness of the oxide film corresponds to $2.1-2.3 \mathrm{~nm}$, respectively. The electrostatic potential impressed across the thin oxide film is then $V \approx-E_{0} L \approx-2.1$ and $-3.5 \mathrm{~V}$, which compare well with the reported values. This results in a significant lowering of activation barriers for ionic diffusions at the metal-oxide and oxide-gas interfaces [shown in Fig. 3(b)], even at temperatures which are otherwise insufficiently high for appreciable thermal diffusion of ions.

In summary, we report one of the first atomistic simulation studies explaining the influence of the electric field on the room temperature oxidation kinetics and ultrathin oxide growth on $\mathrm{Al}$ substrates. The presence of the electric field is found to dramatically increase the kinetics of $\mathrm{Al}$ (100) oxidation, both in the molecular and atomic oxidation environment. The rate of ion migration through the growing oxide film is strongly enhanced, consistent with the Cabrera-Mott theory of low temperature metal oxidation. Increasing the electric field to $\sim 10-15 \mathrm{MV} / \mathrm{cm}$ thus drives the surface chemisorbed oxygen into the oxide interior leading to a dramatic improvement in the stoichiometry and density of the oxide film. It should be noted that additional insights towards understanding the electronic structural changes associated with the surface reactions as well the effect on geometry in the case of electricfield enhanced oxidation can be provided by calculations based on the density function theory $[10,20]$. These will be attempted in the near future.

We thank the Office of Naval Research for providing financial support for this work. S. R. acknowledges Eric Garfunkel for valuable discussions.

*Corresponding author. shriram@deas.harvard.edu

[1] C. L. Chang, M. H. Engelhard, and S. Ramanathan, Appl. Phys. Lett. 92, 263103 (2008).

[2] E. Tan, P. G. Mather, A. C. Perrella, J. C. Read, and R. A. Buhrman, Phys. Rev. B 71, 161401 (2005).

[3] I. Popova, V. Zhukov, and J. T. Yates, Phys. Rev. Lett. 89, 276101 (2002).

[4] V. Zhukov, I. Popova, and J. T. Yates, Phys. Rev. B 65, 6 (2002).

[5] I. Popova, V. Zhukov, and J. T. Yates, Appl. Phys. Lett. 75, 3108 (1999).

[6] I. W. Boyd, V. Craciun, and A. Kazor, Jpn. J. Appl. Phys. 32, 6141 (1993).

[7] C.L. Chang, V. Shutthanandan, S.C. Singhal, and S. Ramanathan, Appl. Phys. Lett. 90, 203109 (2007).

[8] E. T. Byram, T. A. Chubb, and H. Friedman, Phys. Rev. 98, 1594 (1955).

[9] C. L. Chang, S. K. R. S. Sankaranarayanan, M. Engelhard, V. Shutthanandan, and S. Ramanathan, J. Phys. Chem. C 113(9), 3502 (2009).

[10] J.-S. McEwen, P. Gaspard, F. Mittendorfer, T. V.d. Bocarmé, and N. Kruse, Chem. Phys. Lett. 452, 133 (2008).

[11] N. Cabrera and N. F. Mott, Rep. Prog. Phys. 12, 163 (1949).

[12] Timothy J. Campbell et al., Phys. Rev. B 71, 205413 (2005).

[13] F. H. Streitz and J. W. Mintmire, Phys. Rev. B 50, 11996 (1994).

[14] X. W. Zhou, H. N. G. Wadley, J.-S. Filhol, and M.N. Neurock, Phys. Rev. B 68, 065402 (2005).

[15] N. Klein and M. Albert, J. Appl. Phys. 53, 5840 (1982).

[16] A. Hasnaoui, O. Politano, J. M. Salazar, and G. Aral, Phys. Rev. B 73, 035427 (2006).

[17] S. K. R. S. Sankaranarayanan and S. Ramanathan, Phys. Rev. B 78, 085420 (2008).

[18] L. Jeurgens, A. Lyapin, and E. Mittemeijer, Acta Mater. 53, 4871 (2005).

[19] J.L. Erskine and R.L. Strong, Phys. Rev. B 25, 5547 (1982).

[20] D. R. Jennison and A. Bogicevic, Surf. Sci. 464, 108 (2000). 